\title{
Serum Bilirubin Level Predicts Frontal QRS-T Angle Change in Patients with Acute Coronary Syndrome
}

\author{
Güney Erdoğan ${ }^{1}$, Mustafa Yenercağำ, Gündüz Durmus², Diyar Koprulu³ ${ }^{3}$ Uğur Arslan ${ }^{1}$ \\ ${ }^{1}$ Department of Cardiology, University of Health Sciences Turkey, Samsun Training and Research Hospital, Samsun, Turkey \\ ${ }^{2}$ Cardiology Clinic, Sultangazi Haseki Training and Research Hospital, Istanbul, Turkey \\ ${ }^{3}$ Cardiology Clinic, Ordu State Hospital, Ordu, Turkey
}

\section{ABSTRACT}

Introduction: The predictive role of serum bilirubin levels on the alteration of total ischemic burden expressed via frontal QRS-T angle change has never been studied in patients with acute coronary syndrome (ACS). In this study, we aimed to investigate the relationship between serum bilirubin levels and frontal QRS-T angle change after percutaneous coronary intervention (PCI) in ACS patients. Methods: Frontal QRS-T angle change was accepted as positive or negative according to whether the value of the baseline frontal QRS-T angle minus the post PCI frontal QRS-T angle is positive or negative. A total of 314 consecutive patients with ACS who underwent PCI were divided into two groups based on their negative frontal QRS-T angle change ( $n=152$ patients) and positive frontal QRS-T angle change ( $n=162$ patients). Results: Multivariate logistic regression analysis showed that the C-reactive protein (CRP) to albumin ratio (CAR) (Odds ratio [OR]: 0.519, 95\% CI: 0.373-0.724, p <0.001), total bilirubin (OR: 3.687, 95\% CI: 1.151-8.095, p = 0.03), and low-density lipoprotein (LDL) (OR: 0.985, 95\% CI: 0.970-1.000, $\mathrm{p}=0.04$ ) were independent predictors of positive QRS-T angle change. In receiver operating characteristics curve analysis, a cut-off value of 0.59 total bilirubin had a $69 \%$ sensitivity and a $65 \%$ specificity (area under curve: $0.739, \mathrm{p}<0.001$ ) for the prediction of positive QRS-T angle change. Conclusion: In ACS patients, serum total bilirubin has been found to be a useful decision-making tool to predict positive frontal QRS-T angle change as a sign of reduced total ischemic burden, to assess early invasive strategy independently from other study parameters.

Keywords: frontal QRS-T angle, bilirubin, acute coronary syndrome

\section{ARTICLE HISTORY}

Received: September 20, 2020 Accepted: October 31, 2020

\section{CORRESPONDENCE}

Ugur Arslan

Baris Bulvari No: 199

55400 Ilkadim, Samsun, Turkey

Tel: +90 5326039983

Fax: +90 3622778765

E-mail: ugurarslan5@yahoo.com 


\section{INTRODUCTION}

Bilirubin, one of the waste products of heme at first, has strong antioxidant, anti-inflammatory, and antithrombotic effects. ${ }^{1}$ Bilirubin also has anti-inflammatory properties related to the suppression of endothelial adhesion molecules such as intercellular adhesion molecule-1, vascular cell adhesion molecule-1, E-selectin, and tumor necrosis factor-alpha. ${ }^{2}$ However, a small number of studies analyzed the relationship between serum total bilirubin (STB) and the prognosis of percutaneous coronary intervention (PCI) in patients with acute coronary syndrome (ACS), and a certain degree of conflict exists in the reported results. A meta-analysis including a large population-based cohort of 7,222 participants reported that STB was conversely correlated with the risk of cardiovascular diseases. ${ }^{3}$ It has also been demonstrated that in patients with coronary artery disease (CAD), lower STB might represent a predictor of major adverse cardiovascular events after PCI. 4

Frontal QRS-T angle (QRSTa), one of the electrocardiographic parameters to identify high-risk patients, reflects the myocardial depolarization and repolarization heterogeneity, particularly in patients with CAD. ${ }^{5}$ An abnormal frontal QRSTa has been suggested to be closely linked to increased cardiovascular mortality, ${ }^{6}$ and it has been found to predict poor prognosis in patients with ACS. ${ }^{7}$ Raposeiras-Roubin et al. demonstrated that a wide frontal QRSTa $\left(>90^{\circ}\right)$ is a good discriminator of long-term mortality in patients with depressed ejection fraction (EF) after an ACS ${ }^{8}$ while in another study, it has been demonstrated that the postprocedural frontal QRSTa can predict in-hospital mortality and thrombolytic therapy failure. ${ }^{9}$

Investigation on the association of total bilirubin levels with frontal QRSTa, a predictor of general and cardiac mortality, may be beneficial in revealing this issue after PCI in patients with ACS. In the present study, we intended to demonstrate the association between serum bilirubin levels and change of frontal QRSTa after PCI in ACS patients.

\section{METHODS}

\section{STUDY POPULATION}

This study was designed as a cross-sectional single-center study investigating the relationship between STB levels and frontal QRSTa in patients with ACS. A total of 347 consecutive patients with the diagnosis of non-ST-elevation myocardial infarction (NSTEMI) or ST- elevation myocardial infarction (STEMI) who underwent PCI between Decem- ber 2019 and May 2020 were enrolled in the study. STEMI was defined as typical angina with ST-segment elevation of $>0.1 \mathrm{mV}$ in at least two consecutive electrocardiogram (ECG) leads in any leads other than V2-V3. In leads V2-V3, an ST-segment elevation $>0.2 \mathrm{mV}$ was required for STEMI definition. NSTEMI was defined if patients had typical angina pain lasting 30 minutes and a positive troponinI level (defined in our clinical laboratory as $>0.01 \mathrm{ng} / \mathrm{mL}$ ) without any evidence of ST-segment elevation on 12-lead electrocardiogram. Major exclusion criteria included history of congestive heart failure, previous PCI, coronary artery bypass grafting, active infectious disease, inflammatory or immunologic disease, cirrhosis, STB at admission $>2.0 \mathrm{mg} / \mathrm{dL}$, peripheral arterial disease, chronic obstructive pulmonary disease, chronic kidney disease, malignancy, history of blood transfusion in the last 3 months, cardiogenic shock on admission, and the inability to achieve thrombolysis in myocardial infarction (TIMI) 3 flow after PCI. After the recruitment of eligible patients, the study population consisted of 314 patients with the diagnosis of ACS, including 166 NSTEMI and 148 STEMI.

The study was conducted in accordance with the general principles stated in the Declaration of Helsinki, the local research ethics committee reviewed the study protocol, and all patients agreed to participate in the study.

\section{LABORATORY MEASUREMENTS}

Peripheral venous blood ( $3 \mathrm{~mL}$ ) samples were taken from the antecubital vein after the admission of the patients for the measurement of complete blood count (CBC), bilirubin, troponin, liver and kidney function tests, and bleeding profile. Lipid profile and other biochemical parameters were determined using standard techniques on the morning following the admission day in a fasting state of at least 8 hours. Samples for the CBC analysis were collected in EDTA-anticoagulated Monovettew tubes (Sarstedt Monovette, Nuembrecht, Germany). Hemoglobin, white blood cell, neutrophil-lymphocyte ratio (NLR), red cell distribution width (RDW), mean platelet volume, and platelet counts were measured as part of the automated CBC using a Coulter LH 780 Hematology Analyzer (Beckman Coulter Inc, Miami, Florida). An ADVIA1800 automatic biochemical analyzer was used for serum bilirubin level measurements (Siemens, Berlin, Germany). Serum CRP levels were measured with nephelometric method (UniCel DxC 800 System; Beckman Coulter Inc, Brea, California). The CRP to albumin ratio (CAR) was calculated as the ratio of CRP to the albumin level multiplied by 100 . 


\section{DEFINITION OF RISK FACTORS}

The following clinical and demographic parameters were acquired from the medical records: age, gender, hypertension (known hypertension treated with antihypertensive drugs, two or more blood pressure recordings greater than 140/90 $\mathrm{mmHg}$ ), diabetes mellitus (known diabetes treated with diet or drugs or both, or a fasting serum glucose higher than $126 \mathrm{mg} / \mathrm{dL}$ ), hypercholesterolemia (known treated hypercholesterolemia or fasting or nonfasting serum cholesterol concentrations higher than $200 \mathrm{mg} / \mathrm{dL}$ ). Positive family history of CAD was defined as documented evidence of premature $\mathrm{CAD}$ in a close relative (men $<55$ and women $<65$ years of age). Current cigarette smoking was reported as active smoking within the past 12 months. BMI was determined by the following formula: $\mathrm{BMI}=$ weight $(\mathrm{kg}) /$ height2 $(\mathrm{m} 2)$. Cardiogenic shock was defined as obvious and persistent hypotension (>30 min) with systolic arterial pressure $<80 \mathrm{mmHg}$ and signs of hypoperfusion due to the dysfunction of the ventricles of the heart..$^{10}$

\section{PCI PROCEDURE}

All study patients were treated based on the latest evidence-based European Society of Cardiology (ESC) guidelines. ${ }^{11}$ A loading dose of $600 \mathrm{mg}$ of clopidogrel or $180 \mathrm{mg}$ of ticagrelor was given with $300 \mathrm{mg}$ of aspirin once the diagnosis of STEMI or NSTEMI was confirmed. Eligible STEMI patients underwent emergent coronary angiography using the standard Judgkins technique via the femoral or radial artery. The definition of infarctrelated artery was done in different image plans by cine angiography visualization. Primary PCI of the culprit lesion was performed to achieve TIMI 3 flow. In all NSTEMI patients having very high risk criteria, coronary angiography was carried out within $2 \mathrm{~h}$, while the remaining NSTEMI patients were referred to coronary angiography within $24 \mathrm{~h}$. PCI was performed in all appropriate NSTEMI patients. The iodine-based contrast agent (Iopromide; Ultravist-370 Schering AG, Berlin, Germany) was injected manually at each position. PCI of the culprit lesion was performed by a $7 \mathrm{~F}$ guiding catheter to establish TIMI 3 flow. After the administration of 5,000 IU of intravenous heparin $(70 \mathrm{U} / \mathrm{kg})$, direct stenting was performed whenever possible, and in the remaining cases, balloon predilatation was carried out. Additionally, in all patients without contraindications, beta-blockers, angiotensin converting enzyme inhibitors or angiotensin receptor blockers and statin therapy were initiated.

\section{ECG AND ECHOCARDIOGRAPHY}

A basal 12-lead surface ECG (Nihon Kohden Corporation, Cardiofax M Model ECG-1250, Tokyo, Japan) with a $25 \mathrm{~mm} / \mathrm{s}$ paper speed and a voltage of $10 \mathrm{~mm} / \mathrm{s}$ was obtained in supine position, immediately before coronary angiography, and a control ECG was recorded $48 \mathrm{~h}$ after PCI. All of the basal and control ECGs were scanned and transferred to a personal computer to decrease the error measurements and then used at $400 \%$ magnification in Adobe Photoshop software. ECG measurements of QT and Tp-e intervals were conducted by two cardiologists blinded to the patient data. The Tp-e interval was defined as the interval from the peak of the $\mathrm{T}$ wave to the end of the $\mathrm{T}$ wave. The $\mathrm{Tp}$-e intervals were measured from precordial leads. ${ }^{12}$ The QT interval was measured from the beginning of the QRS complex to the end of the T wave. The QTc interval was calculated by using Bazett's formula as QTc interval $=\mathrm{QT} / \sqrt{ }(\mathrm{RR}$ interval $){ }^{13}$ Interobserver and intraobserver agreement values were excellent for both Tp-e and QT intervals (interobserver $\mathrm{r}=0.98, \mathrm{p}<0.001$; intraobserver $\mathrm{r}=0.99, \mathrm{p}<0.001)$. The frontal QRSTa was defined as the absolute value of the difference between the frontal plane $\mathrm{QRS}$ and $\mathrm{T}$ axes. If such a difference was $>180^{\circ}$, QRSTa was adjusted to the minimal angle as $360^{\circ}$ minus the absolute value of the difference between the frontal plane QRS and $\mathrm{T}$ axes. ${ }^{14}$ The subjective component of the individual measurements has been ruled out by calculating frontal QRSTa based on the automatic report of the ECG device. Complete and incomplete right or left bundle branch block (LBBB) or pathological Q wave on a 12-lead ECG were accepted as an exclusion criteria from the study. Frontal QRSTa change was accepted as positive or negative according to whether the value of the baseline frontal QRSa minus the post PCI frontal QRSa was positive or negative. Echocardiography was performed by two experienced cardiologists who were blinded to other data. All measurements were performed in accordance to the latest guidelines. ${ }^{15}$ Left ventricular ejection fraction (LVEF) was quantified according to the biplane modified Simpson's method.

\section{STATISTICAL ANALYSIS}

All statistical calculations were performed using IBM SPSS Statistics for Windows, Version 22.0 (IBM Corp., Armonk, NY, USA). The distribution of continuous variables was tested using the Kolmogorov-Smirnov test. Accordingly, Student's t test or the Mann-Whitney U test were used to compare continuous variables according to the distribu- 
TABLE 1. Comparison of positive QRS-T change and negative QRS-T change groups according to baseline clinical and laboratory features

\begin{tabular}{|c|c|c|c|c|}
\hline & Total & $\begin{array}{l}(+) \text { frontal QRS-T } \\
\text { angle change } \\
n=152\end{array}$ & $\begin{array}{l}\text { (-) frontal QRS-T } \\
\text { angle change } \\
n=162\end{array}$ & $p$ value \\
\hline Age, years & $59.1 \pm 10.1$ & $59.3 \pm 10.4$ & $58.8 \pm 9.8$ & 0.7 \\
\hline Male gender, n (\%) & $142(45.2)$ & $78(48.1)$ & $64(42.1)$ & 0.4 \\
\hline Hypertension, n (\%) & $150(47.8)$ & $70(46.1)$ & $80(49.4)$ & 0.6 \\
\hline Diabetes mellitus, n (\%) & $94(29.9)$ & $34(22.4)$ & $60(37.5)$ & 0.04 \\
\hline Smoking, n (\%) & $134(42.7)$ & $68(44.7)$ & $66(40.7)$ & 0.6 \\
\hline Family history, n (\%) & $182(57.9)$ & $85(55.9)$ & $97(58.6)$ & 0.5 \\
\hline Heart rate, per minute & $80 \pm 17$ & $82 \pm 21$ & $79 \pm 13$ & 0.3 \\
\hline Creatinine, mg/dL & $0.93 \pm 0.31$ & $0.94 \pm 0.37$ & $0.92 \pm 0.24$ & 0.7 \\
\hline $\mathrm{WBC}, \mu \mathrm{L}$ & $11.1 \pm 3.4$ & $10.9 \pm 3.0$ & $11.4 \pm 3.7$ & 0.3 \\
\hline Hemoglobin, g/dL & $13.6 \pm 1.6$ & $13.7 \pm 1.5$ & $13.6 \pm 1.6$ & 0.9 \\
\hline Platelet count, $10^{3} / \mu \mathrm{L}$ & $246 \pm 72$ & $233 \pm 75$ & $261 \pm 68$ & 0.01 \\
\hline NLR & $5.0 \pm 2.9$ & $4.3 \pm 2.7$ & $5.7 \pm 3.1$ & 0.003 \\
\hline Total cholesterol, mg/dL & $209.2 \pm 48.0$ & $195.5 \pm 42.2$ & $209.9 \pm 53.4$ & 0.1 \\
\hline LDL-C, mg/dL & $132.4 \pm 37.7$ & $122.3 \pm 29.9$ & $136.9 \pm 43.5$ & 0.04 \\
\hline HDL-C, mg/dL & $41.4 \pm 12.1$ & $41.6 \pm 12.9$ & $40.7 \pm 10.5$ & 0.7 \\
\hline Triglyceride, mg/dL & $200.1 \pm 111.3$ & $186.5 \pm 93.0$ & $194.6 \pm 112.9$ & 0.7 \\
\hline AST, u/L & $23.6 \pm 8.35$ & $24.1 \pm 9.7$ & $23.5 \pm 7.8$ & 0.7 \\
\hline ALT, u/L & $21.2 \pm 9.3$ & $21.7 \pm 9.9$ & $20.6 \pm 9.2$ & 0.5 \\
\hline Total bilirubin, mg/dL & $0.66 \pm 0.40$ & $0.78 \pm 0.46$ & $0.56 \pm 0.31$ & 0.001 \\
\hline Direct bilirubin, mg/dL & $0.14 \pm 0.11$ & $0.16 \pm 0.14$ & $0.12 \pm 0.06$ & 0.03 \\
\hline Indirect bilirubin, mg/dL & $0.51 \pm 0.32$ & $0.59 \pm 0.35$ & $0.43 \pm 0.28$ & 0.002 \\
\hline $\mathrm{CRP}, \mathrm{mg} / \mathrm{L}$ & $10.2 \pm 6.9$ & $7.3 \pm 4.7$ & $12.9 \pm 7.5$ & 0.001 \\
\hline Albumin, g/L & $38.4 \pm 0.39$ & $39.2 \pm 3.8$ & $37.6 \pm 3.6$ & 0.01 \\
\hline CAR,$\times 100$ & $27.3 \pm 19.1$ & $18.8 \pm 11.5$ & $35.2 \pm 21.4$ & 0.001 \\
\hline STEMI, n (\%) & $148(47.1)$ & $84(56.8)$ & $64(39.5)$ & 0.04 \\
\hline PrePCI frontal QRS-T angle, degrees & $57.5 \pm 47.5$ & $80.2 \pm 47.1$ & $36.2 \pm 37.1$ & 0.001 \\
\hline PostPCI frontal QRS-T angle, degrees & $61.4 \pm 45.2$ & $52.2 \pm 42.1$ & $70.1 \pm 46.6$ & 0.01 \\
\hline Tp-e, msec & $92.5 \pm 21.3$ & $90.4 \pm 19.1$ & $95.1 \pm 23.5$ & 0.3 \\
\hline QT, msec & $390 \pm 26$ & $385 \pm 23$ & $394 \pm 28$ & 0.4 \\
\hline QTc, msec & $423 \pm 39$ & $419 \pm 36$ & $426 \pm 42$ & 0.5 \\
\hline Infarct related artery, n (\%) & & & & 0.5 \\
\hline $\mathrm{LAD}$ & $124(39.4)$ & $64(20.3)$ & $60(19.1)$ & \\
\hline CX & $78(24.8)$ & $32(10.1)$ & $46(14.6)$ & \\
\hline RCA & $112(35.6)$ & $56(17.8)$ & $56(17.8)$ & \\
\hline Multivessel Disease, n (\%) & & & & 0.9 \\
\hline 1-vessel disease & $174(55.4)$ & $86(27.3)$ & $88(28.0)$ & \\
\hline 2-vessel disease & $96(30.5)$ & $44(14.0)$ & $52(16.5)$ & \\
\hline 3-vessel disease & $44(14.0)$ & $22(7.0)$ & $22(7.0)$ & \\
\hline Syntax Score & $11.3 \pm 7.4$ & $10.5 \pm 7.2$ & $12.1 \pm 7.6$ & 0.3 \\
\hline Residual Syntax Score & $4.2 \pm 5.6$ & $3.3 \pm 5.4$ & $4.7 \pm 5.9$ & 0.3 \\
\hline Thrombus, n (\%) & $94(29.9)$ & $28(\% 18.4)$ & $68(42)$ & 0.001 \\
\hline No reflow, \% & 7.9 & 4 & 12 & 0.04 \\
\hline LVEF, \% & $45.3 \pm 6.2$ & $47.1 \pm 6.9$ & $43.7 \pm 4.9$ & 0.001 \\
\hline Troponin I peak, ng/dL & $14.4 \pm 9.1$ & $12.6 \pm 8.4$ & $16.1 \pm 9.3$ & 0.02 \\
\hline
\end{tabular}

CAR - C-reactive protein to albumin ratio; CRP - C-reactive protein; LVEF - left ventricular ejection fraction; WBC - white blood cells; LDL-C - low-density lipoprotein-cholesterol; HDL-C - high-density lipoprotein-cholesterol; NLR - neutrophil to lymphocyte ratio

$\mathrm{p}<0.05$ is statistically significant 
tion of the data. Chi-square or Fisher's exact tests were used to compare categorical variables. Continuous variables were presented as mean $\pm \mathrm{SD}$, whereas categorical variables as count and percentages. Receiver operating characteristic (ROC) analysis was performed to determine the sensitivity and specificity with $95 \%$ confidence intervals (CIs) for total bilirubin and the CAR at cut-off values. Spearman's correlation coefficient was used for correlation analysis. The association of different variables with positive frontal QRSTa change was calculated in univariate analysis. Logistic regression analysis was used to find independent predictors of positive frontal QRSTa change. In this analysis, the variables which differ between groups with a $\mathrm{p}$ value $<0.25$ were included. A p value $<0.05$ was considered to be statistically significant. We calculated the minimum number of individuals that should be sampled with 99\% power and 0.05 Type-I error as at least 133 ( $R$ 3.0.1. open source program).

\section{RESULTS}

The study included 314 patients admitted to the emergency unit with the diagnosis of ACS, including 166 NSTEMI and 148 STEMI patients. Patients were divided into two groups according to frontal QRS-T change polarity. From all patients, 152 had positive QRSTa change, while the remaining 162 patients had negative QRSTa change. Table 1 summarizes the comparison of positive QRSTa change and negative QRSTa change groups according to baseline, clinical, and laboratory features. Diabetes mellitus (DM) ( 34 vs. $60, p=0.04$ ), platelet count ( $233 \pm 75$ vs. $261 \pm 68$, $\mathrm{p}=0.01)$, low-density cholesterol (LDL-C) $(122.3 \pm 29.9$ vs. $136.9 \pm 43.5, \mathrm{p}=0.04)$ levels, neutrophil to lymphocyte ratio (NLR) $(4.3 \pm 2.7$ vs. $5.7 \pm 3.1, p=0.003), \mathrm{CRP}(7.3 \pm$
4.7 vs. $12.9 \pm 7.5, \mathrm{p}<0.001)$, CAR $(7.3 \pm 4.7$ vs. $12.9 \pm 7.5$ p <0.001), thrombus (28 [18.4] vs. 68 [42]; p <0.001), no reflow ( $4 \%$ vs. $12 \%, p=0.04)$, and peak troponin I (12.6 \pm 8.4 vs. $16.1 \pm 9.3, \mathrm{p}=0.002$ ) were lower in the positive QRS-T change group. Total bilirubin ( $0.78 \pm 0.46$ vs. $0.56 \pm$ $0.31, \mathrm{p}<0.001$ ), direct bilirubin ( $0.16 \pm 0.14$ vs. $0.12 \pm 0.06$, $\mathrm{p}=0.03)$, indirect bilirubin $(0.59 \pm 0.35$ vs. $0.43 \pm 0.28 . \mathrm{p}=$ $0.002)$, serum albumin levels ( $39.2 \pm 3.8$ vs. $37.6 \pm 3.6 . p=$ 0.01 ), STEMI ( $84[56.8 \%]$ vs. 64 [39.5\%], p = 0.048), and $\operatorname{LVEF}(47.1 \pm 6.9$ vs. $43.7 \pm 4.9, \mathrm{p}<0.001$ ) were significantly higher among patients with positive QRSTa change compared to patients with negative QRSTa change.

Univariate logistic regression analysis identified that CAR, total bilirubin, NLR, LDL-C, LVEF, platelet count, $\mathrm{DM}$, and peak troponin I were significantly associated with positive QRSTa change in the study population (Table 2). Multivariate logistic regression analysis showed that CAR (OR: 0.519, 95\% CI: 0.373-0.724, p <0.001), total bilirubin (OR: 3.687, 95\% CI: 1.151-8.095, $\mathrm{p}=0.03$ ), and LDL-cholesterol (OR: 0.985, 95\% CI: 0.970-1.000, p $=0.04$ ) were the independent predictors of positive QRSTa change (Table 2).

In the non-STEMI subgroup analysis, univariate logistic regression analysis identified that CAR, LVEF, and peak troponin I were significantly associated with positive QRSTa change. Multivariate logistic regression analysis showed that CAR (OR: 0.622, 95\% CI: 0.400-0.967, $\mathrm{p}=0.03$ ) was the only independent predictor of positive QRSTa change (Table 3).

The correlations between several study parameters are shown in Table 4. Spearman correlation analysis revealed a positive and mild-moderate correlation between total bilirubin and prePCI frontal QRSTa $(r=0.332, \mathrm{p}<0.001)$, and a positive and mild correlation between total bilirubin

TABLE 2. Univariate and multivariate regression analysis of various parameters to predict frontal QRSTa change in the study population

\begin{tabular}{lllllll}
\hline & p value & OR & $\mathbf{9 5 \%} \mathbf{~ C I}$ & $\mathbf{p ~ v a l u e}$ & $\mathbf{~ O R}$ & $\mathbf{9 5 \%} \mathbf{~ C I}$ \\
\hline CAR & 0.001 & 0.567 & $0.4-0.7$ & 0.001 & 0.519 & $0.3-0.7$ \\
Total bilirubin & 0.02 & 1.911 & $1.1-5.1$ & 0.03 & 1.687 & $1.1-4.2$ \\
NLR & 0.004 & 1.621 & $0.7-0.9$ & 0.05 & 0.803 & $0.6-1.006$ \\
LDL & 0.04 & 0.989 & $0.9-1.0$ & 0.04 & 0.985 & $0.9-1.000$ \\
EF & 0.001 & 1.104 & $1.0-1.1$ & 0.1 & 1.070 & $0.9-1.1$ \\
Platelet & 0.02 & 0.994 & $0.9-0.9$ & 0.5 & 0.998 & $0.9-1.005$ \\
DM & 0.04 & 0.480 & $0.2-0.9$ & 0.5 & 1.381 & $0.4-4.1$ \\
Troponin I peak & 0.01 & 0.957 & $0.9-0.9$ & 0.2 & 0.967 & $0.9-1.0$ \\
\hline
\end{tabular}

CAR - C-reactive protein to albumin ratio; CI - confidence interval; DM - diabetes mellitus; EF - ejection fraction; LDL-C - low-density lipoprotein-cholesterol; NLR - neutrophil to lymphocyte ratio 
TABLE 3. Univariate and multivariate regression analysis in the non-STEMI subgroup

\begin{tabular}{lllllll}
\hline & p value & OR & $\mathbf{9 5 \%}$ CI & p value & oR & $\mathbf{9 5 \% ~ C I ~}$ \\
\hline CAR & 0.003 & 0.611 & $0.444-0.842$ & 0.03 & 0.622 & $0.400-0.967$ \\
Total bilirubin & 0.2 & 1.444 & $0.600-3.579$ & 0.2 & 1.387 & $0.418-3.402$ \\
NLR & 0.05 & 0.840 & $0.703-1.003$ & 0.1 & 0.788 & $0.562-1.105$ \\
LDL & 0.7 & 0.997 & $0.982-1.012$ & 0.5 & 0.993 & $0.971-1.015$ \\
EF & 0.001 & 1.264 & $1.124-1.421$ & 0.1 & 1.148 & $0.957-1.378$ \\
Platelet & 0.057 & 0.992 & $0.983-1.000$ & 0.2 & 0.992 & $0.980-1.005$ \\
DM & 0.09 & 0.379 & $0.123-1.172$ & 0.8 & 1.246 & $0.218-4.130$ \\
Troponin I peak & 0.01 & 0.941 & $0.896-0.989$ & 0.2 & 0.956 & $0.883-1.033$ \\
\hline
\end{tabular}

CAR - C-reactive protein to albumin ratio; CI - confidence interval; DM - diabetes mellitus; EF - ejection fraction; LDL-C - low-density lipoprotein-cholesterol; NLR - neutrophil to lymphocyte ratio

and postPCI frontal QRSTa $(\mathrm{r}=0.193, \mathrm{p}=0.016)$ and frontal QRSTa change $(r=0.177, p=0.02)$. The correlation analysis also found a positive and mild correlation between CAR and prePCI frontal QRSTa $(r=0.257, \mathrm{p}<0.001)$, a positive and mild correlation between CAR and postPCI frontal QRSTa $(r=0.175, p<0.03)$, and a positive and mild correlation between CAR and frontal QRSTa change $(r=0.160$, $\mathrm{p}=0.04$ ). In addition, there was a negative and moderate correlation between total bilirubin and CAR $(r=-0.432, p$ $<0.001)$, direct bilirubin and CAR $(r=-0.352, p<0.001)$, and indirect bilirubin and CAR $(r=-0.411, p<0.001)$.

In ROC analysis, a cut-off value of 0.59 total bilirubin had a $69 \%$ sensitivity and a $65 \%$ specificity (area under curve [AUC]: $0.739,95 \%$ CI: $0.663-0.816, p<0.001$ ) for the prediction of positive QRSTa change, while the AUC for CAR was 0.274 (95\% CI: 0.194-0.355, p <0.001) for the prediction of positive QRSTa change.

\section{DISCUSSIONS}

Early diagnosis, identification of high-risk patients, and elucidation of factors that may influence the prognosis of ACS are crucial. Therefore, the early diagnosis and treatment of ACS and the reduction of certain risk indicators are of paramount importance to save the jeopardized myocardium and improve the prognosis of patients with ACS. The role of both frontal QRSTa change and STB in the pathophysiology and prognosis of cardiovascular diseases has been identified in a limited number of related studies, but there is a certain lack of validation studies examining the relationship between these two parameters, which led to the present research. Our study results documented that STB levels were higher in the positive frontal QRSTa change group.

For many years, bilirubin, the end product of heme catabolism, was regarded as a waste product. Heme oxygen-

TABLE 4. Correlations between some study parameters

\begin{tabular}{|c|c|c|c|c|c|c|c|}
\hline & $\begin{array}{l}\text { Total } \\
\text { bilirubin }\end{array}$ & $\begin{array}{l}\text { Direct } \\
\text { bilirubin }\end{array}$ & $\begin{array}{l}\text { Indirect } \\
\text { bilirubin }\end{array}$ & $\begin{array}{l}\text { PrePCI frontal } \\
\text { QRS-T angle }\end{array}$ & $\begin{array}{l}\text { PostPCI } \\
\text { Frontal } \\
\text { QRS-T angle }\end{array}$ & $\begin{array}{l}\text { Frontal } \\
\text { QRS-T angle } \\
\text { change }\end{array}$ & CAR \\
\hline Total bilirubin & & $\begin{array}{l}r=0.554 \\
p<0.001\end{array}$ & $\begin{array}{l}r=0.923 \\
p<0.001\end{array}$ & $\begin{array}{l}r=0.332 \\
p<0.001\end{array}$ & $\begin{array}{l}r=0.193 \\
p=0.01\end{array}$ & $\begin{array}{l}r=0.177 \\
p=0.02\end{array}$ & $\begin{array}{l}r=-0.030 \\
p=0.7\end{array}$ \\
\hline Direct bilirubin & $\begin{array}{l}r=0.554 \\
p<0.001\end{array}$ & & $\begin{array}{l}r=0.394 \\
p<0.001\end{array}$ & $\begin{array}{l}r=0.233 \\
p=0.003\end{array}$ & $\begin{array}{l}r=0.169 \\
p=0.03\end{array}$ & $\begin{array}{l}r=0.159 \\
p=0.04\end{array}$ & $\begin{array}{l}r=-0.109 \\
p=0.1\end{array}$ \\
\hline Indirect bilirubin & $\begin{array}{l}r=0.923 \\
p<0.001\end{array}$ & $\begin{array}{l}r=0.394 \\
p<0.001\end{array}$ & & $\begin{array}{l}r=0.288 \\
p<0.001\end{array}$ & $\begin{array}{l}r=0.173 \\
p=0.03\end{array}$ & $\begin{array}{l}r=0.163 \\
p=0.03\end{array}$ & $\begin{array}{l}r=-0.042 \\
p=0.6\end{array}$ \\
\hline PrePCI frontal QRS-T angle & $\begin{array}{l}r=0.332 \\
p<0.001\end{array}$ & $\begin{array}{l}r=0.233 \\
p=0.003\end{array}$ & & & $\begin{array}{l}r=0.499 \\
p<0.001\end{array}$ & $\begin{array}{l}r=0.529 \\
p<0.001\end{array}$ & $\begin{array}{l}r=-0.076 \\
p=0.3\end{array}$ \\
\hline PostPCI frontal QRS-T angle & $\begin{array}{l}r=0.193 \\
p=0.01\end{array}$ & $\begin{array}{l}r=0.169 \\
p=0.03\end{array}$ & $\begin{array}{l}r=0.173 \\
p=0.03\end{array}$ & $\begin{array}{l}r=0.499 \\
p<0.001\end{array}$ & & $\begin{array}{l}r=-0.391 \\
p<0.001\end{array}$ & $\begin{array}{l}r=0.185 \\
p=0.02\end{array}$ \\
\hline Frontal QRS-T angle change & $\begin{array}{l}r=0.177 \\
p=0.02\end{array}$ & $\begin{array}{l}r=0.104 \\
p=0.1\end{array}$ & $\begin{array}{l}r=0.146 \\
p=0.06\end{array}$ & $\begin{array}{l}r=0.529 \\
p<0.001\end{array}$ & $\begin{array}{l}r=-0.391 \\
p<0.001\end{array}$ & & $\begin{array}{l}r=-0.317 \\
p<0.001\end{array}$ \\
\hline CAR & $\begin{array}{l}r=-0.432 \\
p<0.001\end{array}$ & $\begin{array}{l}r=-0.352 \\
p<0.001\end{array}$ & $\begin{array}{l}r=-0.411 p \\
<0.001\end{array}$ & $\begin{array}{l}r=0.257 p \\
<0.001\end{array}$ & $\begin{array}{l}r=0.175 \\
p<0.03\end{array}$ & $\begin{array}{l}r=0.160 \\
p=0.04\end{array}$ & \\
\hline
\end{tabular}


ase $(\mathrm{HO})$ is a microsomal enzyme that catalyzes the oxidative cleavage of heme. ${ }^{16}$ Under physiologic conditions, HO production in the vascular endothelium is at low levels. ${ }^{16}$ $\mathrm{HO}$ expression is triggered in situations such as increased lipid oxidation, inflammation, and the formation of vascular damage. ${ }^{17}$ Microsomal $\mathrm{HO}$ is the rate-limiting enzyme in the series of reactions and plays a pivotal role in the formation of bilirubin. Of the two isoforms of HO, increased expression of $\mathrm{HO}-1$ in response to various stimuli provides cardioprotection by anti-inflammatory, antiapoptotic, antiproliferative, and antithrombotic effect. ${ }^{18}$ In cases of increased inflammation and stress, such as ACS, HO is induced, and consequently, the bilirubin level is elevated.19 The binding of bilirubin and albumin creates an asymmetric complex, which produces active hydrogen atoms in the molecular structure that can easily bind with oxygen-free radicals, exerting a strong antioxidant effect. Besides, bilirubin can also impede the formation of oxidized low-density lipoprotein (ox-LDL) through its antioxidant effect. ${ }^{20}$ Ollinger et al. suggested that bilirubin can reduce the extent of vascular stenosis by inhibiting the proliferation of vascular smooth muscle cells. ${ }^{21}$ Kuwano et al. also found that a higher STB level reduced the rate of in-stent restenosis after PCI. ${ }^{22}$ A study by Erdogan et al. showed that STB within the upper limit of the normal range is related to better collateral development compared with lower STB in patients with chronic total coronary occlusion, which proves the endothelial function-enhancing effects of bilirubin..$^{23}$ In a study by Yoshino et al., it was also demonstrated that patients with higher serum bilirubin levels have a better endothelial function, which is suggestive of the resistance against the impairment of coronary artery endothelial function. ${ }^{24}$

The frontal QRSTa is a novel risk marker as the absolute value of the difference between frontal $\mathrm{QRS}$ axis and $\mathrm{T}$ axis on 12-lead surface ECG, reflecting myocardial repolarization heterogenity and electrical instability. Briefly, under ischemic conditions, delayed conduction results in depolarization and repolarization heterogeneity, and widening frontal QRSTa occurs. The calculation of spatial QRST is computed, based on software programs, and cannot be derived routinely from daily used ECG devices. ${ }^{5}$ Moreover, a recent study indicated that frontal QRSTa is a suitable clinical alternative for spatial QRSTa in risk prediction. ${ }^{25}$ Therefore, we decided to use frontal QRSTa in this study. Ordinarily, the direction of the myocardial depolarization deflection and repolarization deflection is in the closer orientation. As a result, frontal QRSTa is frequently expected to be a narrow angle $\left(<45^{\circ}\right) .{ }^{26}$ The relationship between frontal QRSTa and the severity of CAD has been demonstrated in previous studies, particularly depend- ing on the number of vessels or localization of lesions. In a previous study involving a larger sample, it was found that the prevalence of 2- or 3-vessel obstructive CAD was significantly higher in patients with a planar QRSTa $>90^{\circ}$ compared to patients with a planar QRSTa $\leq 90^{\circ} .{ }^{27} \mathrm{In}$ addition, it was shown that patients with baseline frontal QRSTa $\geq 95.6^{\circ}$ had significantly higher frequency of 3-vessel disease compared to patients with baseline frontal QRSTa $<95.6^{\circ}$ in STEMI. ${ }^{9}$ Moreover, it was also detected that proximal vessel disease was more frequently encountered in patients with baseline frontal QRSTa $\geq 95.6^{\circ} .{ }^{9}$ In the recent literature, Dogan et al. have indicated that frontal QRSTa is an independent predictor of coronary atherosclerotic burden in STEMI patients. ${ }^{28}$

In our study, we found that total bilirubin levels were significantly higher in the positive frontal QRSTa change group. Moreover, low rise in bilirubin level may occur as a result of inflammation in some patients, due to biochemical and genetic variations in heme metabolism. Thus, it can be regarded that the HO pathway is less induced following NSTEMI because the extent of the ischemic injury and tissue damage is lower compared to patients with STEMI. It may be a plausible explanation for the lack of an association of this kind between total bilirubin levels and the presence of positive frontal QRSTa change in patients with NSTEMI. Besides, in our study, patients admitted with cardiogenic shock were also excluded. Despite the fact that LVEF was found to be the most important indicator to reflect infarct size, one of the most important results of our study is that a higher STB level was shown to be a powerful predictor of positive frontal QRSTa change as a sign of lower residual ischemia, independently from LVEF. Consequently, the extent of myocardial damage and scar tissue resulting from thrombus and no-reflow may be larger, and the rate of negative frontal QRSTa change as a sign of greater residual ischemia may increase in patients with low bilirubin levels independently from SYNTAX and residual SYNTAX score. In the present study, there was a significant negative correlation between the levels of total bilirubin and CAR values. This result suggests that reduced STB indicates the presence of an inflammatory response as the possible reason for the incidence and mortality of CAD, as shown in a previous study. ${ }^{29}$ This important result of our study supports the beneficial effects of high bilirubin levels on the cardiovascular system.

\section{LIMITATIONS}

First, this was a cross-sectional study which could not infer a causal relation and included a relatively small num- 
ber of patients from a single center. Furthermore, the bilirubin level is a dynamic parameter and could be different from day to day. Therefore, analyses based on a single measurement may not reflect the long-term relationship between bilirubin level and the presence of frontal QRSTa change. The lack of cardiovascular outcomes, including re-intervention rates and mortality, is another limitation. Although we used a multivariable model to adjust for potential confounders, there may remain unmeasured or residual confounding because of the limited sample size. In addition, patients with LBBB were excluded because LBBB is a confounder factor to measure frontal QRSTa, and in several previous studies regarding frontal QRSTa, LBBB was also excluded. ${ }^{30} \mathrm{~A}$ separate study including only patients with LBBB may be conducted to investigate frontal QRSTa in ACS. To clarify the pathophysiological mechanisms and clinical outcomes, prospective large-scale studies are needed.

\section{CONCLUSION}

In patients with ACS, STB predicts admission ischemia, residual ischemia, and dynamic alteration of ischemic burden after PCI independently from other study parameters, as demonstrated by frontal QRSTa change in patients with ACS. In addition, higher STB was also negatively associated with inflammation as demonstrated by CAR.

\section{CONFLICT OF INTEREST}

Nothing to disclose.

\section{REFERENCES}

1. Sarady-Andrews JK, Liu F, Gallo D, et al. Biliverdin administration protects against endotoxin-induced acute lung injury in rats. Am J Physiol Lung Cell Mol Physiol. 2005;289:L1131-L1137. doi: 10.1152/ajplung.00458.2004.

2. NishimuraT, Tanaka M, Saisho Y, Miyakoshi K, Tanaka M, Itoh $\mathrm{H}$. Lower serum total bilirubin concentration is associated with higher prevalence of gestational diabetes mellitus in Japanese pregnant women. Endocr J. 2018;65:1199-1208. doi: 10.1507/endocrj.EJ17-0533.

3. Yip HK, Chen MC, Chang HW, et al. Angiographic morphologic features of infarct-related arteries and timely reperfusion in acute myocardial infarction: predictors of slow-flow and no-reflow phe-nomenon. Chest. 2002;122:1322-1332. doi: 10.1378/chest.122.4.1322.

4. Xu Y, Qu X, Fang W, Chen H. Prevalence, correlation and clinical outcome of intra-procedural stent thrombosis in patients undergoing primary percutaneous coronary intervention for acute coronary syndrome. J Interv Cardiol. 2013;26:215-220. doi: 10.1111/joic.12029.
5. Oehler A, Feldman T, Henrikson CA, Tereshchenko LG. QRS-T angle: a review. Ann Noninvasive Electrocardiol. 2014;19:534542. doi: 10.1111/anec.12206.

6. Zhang, ZM, Prineas, RJ, Case D, Soliman EZ, Rautaharju PM. Comparison of the prognostic significance of the electrocardiographic QRS/T angles in predicting incident coronary heart disease and total mortality (from the atherosclerosis risk in communities study). Am J Cardiol. 2007;100:844-849. doi: 10.1016/j.amjcard.2007.03.104.

7. Lown MT, Munyombwe T, Harrison W, et al. Association of frontal QRS-T angle-age risk score on admission electrocardiogram with mortality in patients admitted with an acute coronary syndrome. Am J Cardiol. 2012;109:307-313. doi: 10.1016/j.amjcard.2011.09.014.

8. Roubin SR, Lamela AV, Cruz NB, et al. Usefulness of the QRS-T angle to improve long-term risk stratification of patients with acute myocardial infarction and depressed left ventricular ejection fraction. Am J Cardiol. 2014;113:1312-1319. doi: 10.1016/j.amjcard.2014.01.406.

9. Colluoglu T, Tanriverdi Z, Unal B, Ozcan EE, Dursun H, Kaya D. The role of baseline and post-procedural frontal plane QRS-T angles for cardiac risk assessment in patients with acute STEMI. Ann Noninvasive Electrocardiol. 2018;23:e12558. doi: 10.1111/anec.12558.

10. Yao HM, Wan YD, Zhang XJ, et al. Long-term follow-up results in patients undergoing percutaneous coronary intervention (PCI) with drug-eluting stents: results from a single highvolume PCI centre. BMJ Open. 2014;4:e004892. doi: 10.1136/ bmjopen-2014-004892.

11. Roffi M, Patrono C, Collet JP, et al. 2015 ESC Guidelines for the management of acute coronary syndromes in patients presenting without persistent ST-segment elevation: Task Force for the Management of Acute Coronary Syndromes in Patients Presenting without Persistent ST-Segment Elevation of the European Society of Cardiology (ESC). Eur Heart J. 2016;37:267-315. doi: 10.1093/eurheartj/ehv320.

12. Hevia JC, Antzelevitch C, Barzaga FT, et al. Tpeak-Tend and Tpeak-Tend dispersion as risk factors for ventricular tachycardia/ventricular fibrillation in patients with the Brugada syndrome. J Am Coll Cardiol. 2006;47:1828-1834. doi: 10.1016/j.jacc.2005.12.049.

13. Goldenberg I, Moss AJ, Zareba W. QT interval: how to measure it and what is "normal". J Cardiovasc Electrophysiol. 2006;17:333-336. doi: 10.1111/j.1540-8167.2006.00408.x.

14. Oehler A, Feldman T, Henrikson CA, Tereshchenko LG. QRS-T angle: a review. Ann Noninvasive Electrocardiol. 2014;19:534542. doi: 10.1111/anec.12206.

15. Lang RM, Badano LP, Avi VM, et al. Recommendations for cardiac chamber quantification by echocardiography in adults: an update from the American Society of Echocardiography and the European Association of Cardiovascular Imaging. J Am Soc Echocardiogr. 2015;28:1-39. doi: 10.1093/ehjci/jew041.

16. Fredenburgh LE, Merz AA, Cheng S. Haeme oxygenase signalling pathway: implications for cardiovascular disease. Eur Heart J. 2015;36:1512-1518. doi: 10.1093/eurheartj/ehv114.

17. Ryter SW, Alam J, Choi AM. Heme oxygenase-1/ carbon monoxide: from basic science to therapeutic applications. Physiol Rev. 2006;86:583-650. doi: 10.1152/ physrev.00011.2005.

18. Fredenburgh LE, Merz AA, Cheng S. Haemeoxygenase signalling pathway: Implications for cardiovascular disease. 
Eur Heart J. 2015;36:1512-1518. doi: 10.1093/eurheartj/ehv114.

19. Okuhara K, Kisaka T, Ozono R, et al. Change in bilirubin level following acute myocardial infarction is an index for heme oxygenase activation. South Med J. 2010;103:876-881. doi: 10.1097/SMJ.ob013e3181eac06a.

20. Daub K, Seizer P, Stellos K, et al. Oxidized LDL-activated platelets induce vascular inflammation. Semin Thromb Hemost. 2010;36:146-156. doi: 10.1055/s-0030-1251498.

21. Ollinger R, Bilban M, Erat A, et al. A natural inhibitor of vascular smooth muscle cell proliferation. Circulation. 2005;112:10301039. doi: 10.1161/CIRCULATIONAHA.104.528802.

22. Kuwano T, Miura SI, Shirai K, et al. Serum levels of bilirubin as an independent predictor of coronary in-stent restenosis: a new look at an old molecule. J Atheroscler Thromb. 2011;18:574-583. doi: 10.5551/jat.6643.

23. Erdogan T, Cicek Y, Kocaman SA, et al. Increased serum bilirubin level is related to good collateral development in patients with chronic total coronary occlusion. Intern Med. 2012;51:249-255. doi: 10.2169/internalmedicine.51.6417.

24. Yoshino S, Hamasaki S, Ishida S, et al. Relationship between bilirubin concentration, coronary endothelial function, and inflammatory stress in overweight patients. J Atheroscler Thromb. 2011;18:403-412. doi: 10.5551/jat.6346.

25. Zhang ZM, Rautaharju PM, Prineas RJ, Tereshchenko LG, Soliman EZ. Electrocardiographic QRS-T angle and the risk of incident silent myocardial infarction in the Atherosclerosis Risk in Communities study. J Electrocardiol. 2017;50:661-666. doi: 10.1016/j.jelectrocard.2017.05.001.

26. Gungor M, Celik M, Yalcinkaya E, et al. The value of frontal planar QRS-T angle in patients without angiographically apparent atherosclerosis. Med Princ Pract. 2017;26:125-131. doi: 10.1159/000453267.

27. Palaniswamy C, Singh T, Aronow WS, et al. A planar QRS-T angle $>90$ degrees is associated with multivessel coronary artery disease in patients undergoing coronary angiography. Med Sci Monit. 2009;15:31-34.

28. Dogan A, Kahraman S. Frontal QRS-T Angle Predicts Coronary Atherosclerotic Burden in Patients With ST Segment Elevation Myocardial Infarction. J Electrocardiol. 2019;58:155-159. doi: 10.1016/j.jelectrocard.2019.11.042.

29. Inoue $\mathrm{T}$, Sonoda $\mathrm{N}$, Hiramatsu S, Kimura S, Ogawa $\mathrm{Y}$, Inoguchi T. Serum bilirubin concentration is associated with left ventricularremodeling in patients with type 2 diabetes mellitus: a cohort study. Diabetes Ther. 2018;9:331-338. doi: 10.1007/s13300-018-0368-6.

30. Kahraman S, Kalkan AK, Turkyilmaz AB, et al. Frontal QRS-T angle is related with hemodynamic significance of coronary artery stenosis in patients with single vessel disease. Anatol J Cardiol. 2019;22:194-201. doi: 10.14744/ AnatolJCardiol.2019.99692. 